\title{
Fast digestive, leucine-rich, soluble milk proteins improve muscle protein anabolism, and mitochondrial function in undernourished old rats
}

\author{
Jérôme Salles ${ }^{1}$, Audrey Chanet ${ }^{1}$, Alexandre Berry ${ }^{1}$, Christophe Giraudet ${ }^{1}$, Véronique Patrac ${ }^{1}$, \\ Carla Domingues-Faria ${ }^{1}$, Christophe Rocher ${ }^{2}$, Christelle Guillet ${ }^{1}$, Philippe Denis ${ }^{1}$, \\ Corinne Pouyet ${ }^{1}$, Cécile Bonhomme ${ }^{3,4}$, Pascale Le Ruyet ${ }^{3,4}$, Yves Rolland ${ }^{5,6}$, Yves Boirie ${ }^{1,7}$ \\ and Stéphane Walrand ${ }^{1}$ \\ ${ }^{1}$ Université Clermont Auvergne, INRA, UNH, Unité de Nutrition Humaine, Clermont-Ferrand, France \\ 2 UMR 5200, CNRS, Université de Bordeaux, Villenave d'Ornon, France \\ ${ }^{3}$ Lactalis Nutrition Santé, Torcé, France \\ ${ }^{4}$ Lactalis Research and Development, Retiers, France \\ ${ }^{5}$ Gérontopôle de Toulouse, Institut du Vieillissement Centre Hospitalo-Universitaire de Toulouse (CHU Toulouse), \\ Toulouse, France \\ ${ }^{6}$ UMR INSERM 1027, University of Toulouse III, Toulouse, France \\ ${ }^{7} \mathrm{CHU}$ Clermont-Ferrand, Clinical Nutrition Department Clermont-Ferrand, France
}

Scope: One strategy to manage malnutrition in older patients is to increase protein and energy intake. Here, we evaluate the influence of protein quality during refeeding on improvement in muscle protein and energy metabolism.

Methods and results: Twenty-month-old male rats $(n=40)$ were fed $50 \%$ of their spontaneous intake for 12 weeks to induce malnutrition, then refed ad libitum with a standard diet enriched with casein or soluble milk proteins (22\%) for 4 weeks. A 13C-valine was infused to measure muscle protein synthesis and expression of MuRF1, and MAFbx was measured to evaluate muscle proteolysis. mTOR pathway activation and mitochondrial function were assessed in muscle. Malnutrition was associated with a decrease in body weight, fat mass, and lean mass, particularly muscle mass. Malnutrition decreased muscle mTOR pathway activation and protein FSR associated with increased MuRF1 mRNA levels, and decreased mitochondrial function. The refeeding period partially restored fat mass and lean mass. Unlike the casein diet, the soluble milk protein diet improved muscle protein metabolism and mitochondrial function in old malnourished rats.

Conclusions: These results suggest that providing better-quality proteins during refeeding may improve efficacy of renutrition in malnourished older patients.

Keywords:

Malnutrition / Old rats / Refeeding / Skeletal muscle / Soluble milk proteins

\section{Introduction}

The chronic diseases and the physiological and psychosocial changes that occur in aging put older adults at higher nutritional risk, both during health service admissions and while in the community $[1,2]$. The malnutrition arises from a combi-

Correspondence: Dr. Stéphane Walrand

E-mail: stephane.walrand@inra.fr

Abbreviations: AA, amino acids; BCAA, branched-chain amino acids; ILE, isoleucine; LEU, leucine; VAL, valine
Received: March 27, 2017

Revised: July 3, 2017

Accepted: July 20, 2017 
resulting in dramatic body weight loss and deregulation of protein metabolism in terms of nitrogen balance and protein content, especially at skeletal muscle level [6]. Muscle is therefore one the prime targets of malnutrition [7]. The mechanisms underpinning muscle depletion in malnourished older subjects are not fully understood but almost certainly involve anomalies in tissue protein and energy metabolism. It was recently proposed [8] that the basis for the inhibition of protein synthesis under such conditions is likely to be multifactorial and includes lack of amino acids (AA), insulin/insulin-like growth factor 1 resistance, proinflammatory cytokine expression, corticosteroids, and/or physical inactivity. In summary, malnutrition increases the risk of morbidity and mortality in older people, particularly due to accelerated muscle loss, but refeeding appears to have less effectiveness at this age of life.

It was previously reported that older malnourished outpatients show lower response, i.e. increase in body weight and blood nutritional markers, to enteral nutrition compared to younger patients [9]. Resistance to renutrition is multifactorial but seems mainly due to the significant increase in AA catabolism within the splanchnic area that occurs in the elderly $[10,11]$ and in aged rats [12]. The high splanchnic extraction of AA during aging might explain the higher intestinal response to refeeding, particularly the increase in enterocyte proliferation, and the subsequent lower plasma AA concentrations observed in old refed rats compared to younger rats [13-16]. Consequently, although intestine structure is restored, the lower availability of nutrients for peripheral tissues may contribute to the poor anabolic responses during refeeding, i.e. lack of increase in muscle mass and protein content, in old malnourished rats [16]. However, supplying a very high, i.e. $27 \%$, protein diet to malnourished old rats saturated AA utilization in visceral tissues and thus enabled a replenishment of the plasma pool of AA. A very-highprotein diet could thereby allow higher AA impregnation of peripheral tissues and thus improve muscle mass in old animals [16]. Malnourished elderly people may therefore require higher amounts of protein during refeeding, possibly due to increased protein utilization by splanchnic tissues.

Not only quantity but also quality of protein intake is an important factor to be considered. Milk protein fractions have been used as a therapeutic modality to conserve muscle mass in chronic wasting diseases and malnutrition. The two major bovine milk protein fractions, i.e. soluble milk proteins and caseins, have quite different patterns of digestion. Soluble milk proteins remain in a liquid form in the stomach, and thus strongly increase AA availability as they are rapidly absorbed through the gut, whereas with slow proteins like caseins that clot at low $\mathrm{pH}$ in the stomach, the increase in AA availability is lower. The resulting magnitude and duration of changes in AA availability determine the anabolic effects of the protein. Only faster digesting proteins (and not the slow proteins) were able to promote whole-body anabolism in elderly subjects. As we recently showed in a doseresponse study $[17,18]$, fast proteins can overcome splanchnic tissue extraction whereas slow proteins are unable to escape it.
Soluble milk proteins are rich in branched-chain amino acids (BCAA), including leucine (LEU), isoleucine (ILE), and valine (VAL). The use of soluble milk proteins was found to increase plasma BCAA concentrations, suggesting a better availability of LEU for peripheral tissues leading to a greater net LEU balance in the postprandial state than when casein was used. As excess LEU has been demonstrated to overcome the agerelated resistance to muscle protein synthesis [19], nutritional manipulation to increase LEU availability could be beneficial in maintaining the postprandial stimulation of protein synthesis during malnutrition in older patients.

Consequently, improving protein quality by combining digestion rate and LEU content may be a more attractive option than simply increasing the quantity of protein intake or the quantity of free LEU to improve protein retention in older patients suffering from protein-energy malnutrition. We thus investigated whether malnutrition-induced alterations, in terms of muscle protein and energy metabolism, were reversible in old rats receiving renutrition via a casein- or soluble milk protein-enriched diet. More precisely, we measured the rate of protein synthesis and the expression of two E3 ligases in skeletal muscle, as these two enzymes are often associated with an activation of the atrophy process in skeletal muscle [20]. As mitochondria play a pivotal role in aerobic ATP production, particularly in muscle cells, we also measured mitochondrial enzyme activities. We hypothesized that the efficacy of protein intake during refeeding is influenced by protein quality, and that soluble milk protein would induce a better response to renutrition than casein in old malnourished rats.

\section{Methods}

\subsection{Animals and experimental design}

All rats and experimental procedures were used in accordance with Clermont-Ferrand University (France) IRB guidelines, and this animal study was approved by the local ethics committee (permission number C2EA-02). Forty old male Wistar rats were purchased from Janvier (Le Genest-St-Isle, France). All rats were the same age at the end of the study, i.e. 23 months old at the end of the dietary restriction period and 24 months old at the end of the refeeding period. All rats were from the same batch and bred under the same conditions throughout their lives. The rats were housed in individual cages under a $12 \mathrm{~h} / 12 \mathrm{~h}$ light/dark cycle (lights on at 8:00 a.m.) at $22^{\circ} \mathrm{C}$. Spontaneous intakes of a control diet were calculated in week two of the acclimatization period (Table 1). old rats consumed $22.6 \mathrm{~g}$ food/day.

\subsubsection{Dietary restriction}

Thirty old rats were fed $50 \%$ (11.3 g/day) of their spontaneous intake for 12 weeks. 
Table 1. Diet composition and amino acid content

\begin{tabular}{|c|c|c|c|}
\hline & Control C & CAS $\mathrm{P}$ & PRO \\
\hline \multicolumn{4}{|l|}{ Diet composition $(\mathrm{g} / 100 \mathrm{~g})$} \\
\hline \multicolumn{4}{|l|}{ Protein } \\
\hline Casein & 14 & 22 & 0 \\
\hline Soluble milk proteins & 0 & 0 & 22 \\
\hline Fat & 4 & 6 & 6 \\
\hline Starch & 46 & 46 & 46 \\
\hline Sucrose & 28 & 18 & 18 \\
\hline Cellulose & 4 & 4 & 4 \\
\hline Vitamin and mineral mix & 4 & 4 & 4 \\
\hline Total energy (kcal/100g) & 404 & 414 & 414 \\
\hline \multicolumn{4}{|l|}{ Amino acid content ( $\mathrm{g} / 100 \mathrm{~g}$ protein) } \\
\hline Isoleucine & 4.92 & 4.92 & 5.63 \\
\hline Leucine & 8.33 & 8.33 & 10.4 \\
\hline Methionine & 3.21 & 3.21 & 2.12 \\
\hline Phenylalanine & 4.86 & 4.86 & 3.33 \\
\hline Tyrosine & 5.03 & 5.03 & 2.78 \\
\hline Valine & 6.82 & 6.82 & 6.07 \\
\hline Lysine & 7.39 & 7.39 & 8.32 \\
\hline Cysteine & 0.41 & 0.41 & 2.60 \\
\hline Threonine & 3.67 & 3.67 & 6.70 \\
\hline Tryptophan & 1.07 & 1.07 & 1.55 \\
\hline Arginine & 3.50 & 3.50 & 2.67 \\
\hline Histidine & 2.77 & 2.77 & 1.82 \\
\hline Alanine & 2.84 & 2.84 & 4.95 \\
\hline Aspartic acid & 6.44 & 6.44 & 10.4 \\
\hline Glutamic acid & 21.7 & 21.7 & 17.1 \\
\hline Glycine & 1.74 & 1.74 & 2.05 \\
\hline Proline & 10.2 & 10.2 & 6.20 \\
\hline Serine & 5.02 & 5.02 & 5.25 \\
\hline Essential amino acids & 48.4 & 48.4 & 51.3 \\
\hline
\end{tabular}

\subsubsection{Refeeding}

At the end of the dietary restriction period, the old rats were randomized into three groups. One group was sacrificed (dietrestricted rats) and the other two rat groups were refed for 4 weeks with a diet containing either $22 \%$ casein (CAS diet) or $22 \%$ soluble milk proteins, i.e. PROLACTA ${ }^{\circledR}$ (PRO diet) (Table 1). The protein we used, i.e. PRO, is a soluble milk protein directly extracted from milk. Unlike whey protein, this constant composition protein is manufactured via a membrane process carried out at low temperature (no protein denaturation, preserved biological activities).

\subsubsection{Control rats}

Ten old rats were fed the control diet ad libitum during the 3-month dietary restriction period, and were then sacrificed.

On the day of sacrifice, rats were weighed and killed by decapitation under anesthesia. Liver, heart, and hindlimb skeletal muscles were weighed, immediately frozen in liquid nitrogen, and stored at $-80^{\circ} \mathrm{C}$ for later measurements. The plantaris muscle was chosen for most muscle analysis as it is a preferentially type- 2 muscle, whose metabolism and contractile functions are changed during aging and undernutrition.

\subsection{Whole body composition analysis}

At the beginning and end of each dietary period, rats were placed in an EchoMRI-100 analyzer (Echo Medical Systems LLC, Houston, TX) to determine fat and lean body mass (g).

\subsection{Dosage of blood insulin}

Fasting plasma insulin concentration was measured by ELISA (MyBioSource, San Diego, CA).

\subsection{Protein synthesis measurement}

To study muscle protein synthesis, we measured rate of incorporation of a stable isotope, i.e. a labeled AA (L-[1-13C] valine, Eurisotop Saint-Aubin, France) into muscle proteins using the flooding dose method. After an overnight fast, rats were injected subcutaneously with a large dose of L-[1-13C] valine $(50 \% \mathrm{~mol}$ excess, $300 \mu \mathrm{M} / 100 \mathrm{~g})$ to flood the precursor pool of protein synthesis. Tracer incorporation time was $50 \mathrm{~min}$ in all groups. A 200-mg piece of plantaris was used to isolate mixed, mitochondrial, myofibrillar, and sarcoplasmic proteins as previously described $[21,22]$. In these studies, the purity of the mitochondrial fraction was determined by measuring percent oligomycin-insensitive ATPase activity as a ratio of total activity, i.e. a measure of contamination with nonmitochondrial membrane structures. Oligomycin inhibited total muscle ATPase activity by more than $90 \%$ in the mitochondria isolated from muscle, indicating only minor contamination of the mitochondrial pellet by nonmitochondrial fractions. After protein hydrolysis, AA were derivatized, and L[13-C]valine enrichment in hydrolyzed proteins was measured by gas chromatography-combustion-isotope ratio mass spectrometry (Gas System; Fisons Instruments, VG Isotech, Middlewich, UK). L[1-13C]valine enrichments in tissue fluid were assessed using gas chromatography-mass spectrometry (Hewlett-Packard 5971A; Hewlett-Packard Co., Palo Alto, CA) and used as precursor pool enrichment to calculate the fractional synthesis rates (FSR).

FSR of proteins were calculated using the following equation:

$\mathrm{FSR}=(\mathrm{Ei} \times 100) /(\mathrm{Ep} \times t)$

where Ei is enrichment as atom percent excess of L-[1$13 \mathrm{C}$ ]valine derived from valine from proteins at time $t$ (minus basal enrichment), Ep is mean enrichment in the precursor pool (tissue fluid L-[1-13C]valine), and t is incorporation time in hours. Data are expressed as \% of control group value or $\%$ of diet-restricted group value. 


\subsection{Western blot analysis}

Fifty milligrams of frozen plantaris was minced and homogenized in an ice-cold buffer $(50 \mathrm{mM}$ HEPES $\mathrm{pH}$ 7.4, $150 \mathrm{mM} \mathrm{NaCl}, 10 \mathrm{mM}$ EDTA, $10 \mathrm{mM}$ NaPPi, $25 \mathrm{mM}$ b-glycerophosphate, $100 \mathrm{mM}$ sodium fluoride, $2 \mathrm{mM} \mathrm{Na}$ orthovanadate, $10 \%$ glycerol, $1 \%$ Triton X-100) containing $1 \%$ protease inhibitor cocktail (Sigma-Aldrich, Saint-QuentinFallavier, France). Homogenates were centrifuged at 12000 $\mathrm{g}$ for $10 \mathrm{~min}$ at $4^{\circ} \mathrm{C}$. Fifty micrograms of denaturated proteins was separated by SDS-PAGE on a polyacrylamide gel and transferred to a polyvinylidene membrane (Millipore, France). Immunoblots were blocked with TBS-Tween-20 $0.1 \%$ containing $5 \%$ dry milk, and then probed with primary antibodies: anti-phospho eIF2 $\alpha$ (Ser51), anti-total eIF2 $\alpha$, antiphospho p70 S6 Kinase (Thr389), anti-total p70 S6 Kinase, anti-phospho 4EBP1 (Ser65), and anti-total 4EBP1 (Cell Signaling Technology, from Ozyme, Saint-Quentin-en-Yvelines, France). After several washes with TBS plus 0.1\% Tween-20, immunoblots were incubated with a horseradish peroxidaseconjugated secondary antibody (DAKO, Trappes, France). Luminescent visualization of secondary antibodies was done using ECL Western Blotting Substrate (Pierce, Thermo Fisher Scientific, Courtaboeuf, France) and an MF-ChemiBIS 2.0 imaging system (F.S.V.T., Courbevoie, France). Band densities were quantified using MultiGauge 3.2 software (Fujifilm Corporation). Phosphorylation states were evaluated based on ratio of phosphorylated protein-to-total protein expression.

\subsection{RNA and DNA analysis}

Total RNA was extracted from 50 mg of frozen plantaris using Tri-Reagent according to the manufacturer's instructions (Euromedex, Mundolsheim, France). RNA was quantified by measuring optical density at $260 \mathrm{~nm}$. Concentrations of $\mathrm{mR}$ NAs corresponding to genes of interest were measured by reverse transcription followed by PCR using a Rotor-Gene $\mathrm{Q}$ system (Qiagen, Courtaboeuf, France). Five micrograms of total RNA was reverse-transcribed using SuperScript III reverse transcriptase and a combination of random hexamer and oligo dT primers (Invitrogen, Life Technologies, SaintAubin, France). cDNAs were diluted 1:60 before PCR analysis. PCR amplification was performed in a $10 \mu \mathrm{L}$ total reaction volume. The PCR mixture contained $2.5 \mu \mathrm{L}$ diluted cDNA template, $5 \mu \mathrm{L} 2 \times$ Rotor-Gene SYBR Green PCR master mix (Qiagen, Courtaboeuf, France), and $0.5 \mu \mathrm{M}$ forward and reverse primers. The amplification profile was initiated by a 5-min incubation at $95^{\circ} \mathrm{C}$ to activate HotStarTaq Plus DNA Polymerase, followed by 40 cycles of two steps: $95^{\circ} \mathrm{C}$ for $5 \mathrm{~s}$ (denaturation step) and $60^{\circ} \mathrm{C}$ for $10 \mathrm{~s}$ (annealing/extension step). Relative mRNA concentrations were analyzed using Rotor-Gene software. Sevenfold serial dilutions from a mix of all undiluted cDNA were used for each target gene to construct linear standard curves from which the concentrations of the test sample were calculated. Table 2 lists the primers used for quantitative real-time PCR amplification. Involvement of the ubiquitin-proteasome pathway in the loss of skeletal muscle mass in undernourished old rats was assessed by measuring MAFbx and MuRF1 gene expression in plantaris muscle. mRNA levels were normalized to a geometric mean of beta-actin, Polr2a, and CypA mRNA expression values [23].

Nuclear/mitochondrial DNA was isolated using TriReagent followed by back-extraction with $4 \mathrm{M}$ guanidine thiocyanate, $50 \mathrm{mM}$ sodium citrate, and $1 \mathrm{M}$ Tris, and an alcohol precipitation step. Mitochondrial DNA (mtDNA) content was determined by quantitative real-time PCR analysis using a Rotor-Gene Q system (Qiagen, Courtaboeuf, France). To this end, the levels of NADH dehydrogenase subunit 1 (ND1) (mitochondrial DNA) were normalized to the levels of betaactin (genomic DNA). The primer sequences are reported in Table 2.

\subsection{Mitochondrial enzymatic assays}

Fifty micrograms of frozen plantaris was homogenized with a glass-glass Potter in nine volumes of homogenization buffer (225 mM mannitol, $75 \mathrm{mM}$ sucrose, $10 \mathrm{mM}$ Tris- $\mathrm{HCl}, 10 \mathrm{~m}$ EDTA, pH 7.2) and spun down at $650 \mathrm{~g}$ for $20 \mathrm{~min}$ at $4^{\circ} \mathrm{C}$. The supernatant was kept and the pellet was suspended in nine volumes of homogenization buffer then submitted to the same procedure. Both supernatants were pooled and used for the assays [24-26].

\subsubsection{Complex II activity}

The assay was performed by following the decrease in absorbance at $600 \mathrm{~nm}$ resulting from the reduction of 2,6dichlorophenolindo-phenol (DCPIP) in $1 \mathrm{~mL}$ of medium containing $50 \mathrm{mM} \mathrm{KH}_{2} \mathrm{PO}_{4}$ (pH 7.5), $1 \mathrm{mM} \mathrm{KCN}, 2 \mu \mathrm{g} / \mathrm{mL}$ rotenone, $20 \mathrm{mM}$ succinate, $100 \mu \mathrm{M}$ ATP, $100 \mu \mathrm{M}$ DCPIP, and $40 \mu \mathrm{g}$ muscle supernatant proteins at $37^{\circ} \mathrm{C}$. The reaction was initiated by adding $100 \mu \mathrm{M}$ decylubiquinone. The absorbance change was monitored for $5 \mathrm{~min}$. Complex II activities were expressed in $\mathrm{nmol} / \mathrm{min} / \mathrm{mg}$ proteins.

\subsubsection{Citrate synthase activity}

Citrate synthase activity was measured by following, at 412 $\mathrm{nm}$, the chemical reduction of 5,5-dithiobis(2-nitrobenzoic acid) (DTNB) by CoASH, which is generated in the citrate synthesis reaction. The reaction mixture contained $200 \mathrm{mM}$ Tris-HCl (pH 8.0), $300 \mu \mathrm{M}$ acetyl-CoA, $150 \mu \mathrm{M}$ DTNB, and $20 \mu \mathrm{g}$ of muscle supernatant proteins at $37^{\circ} \mathrm{C}$. The reaction was initiated by adding $500 \mu \mathrm{M}$ oxaloacetate. The absorbance change was monitored for $3 \mathrm{~min}$. Citrate synthase activities were expressed in $\mathrm{nmol} / \mathrm{min} / \mathrm{mg}$ proteins. 
Table 2. Primer sequences for the quantitative analysis of gene expression and mtDNA content

\begin{tabular}{|c|c|}
\hline Gene name & Forward and reverse primers \\
\hline MAFbx & For 5'- AGTGAAGACCGGCTACTGTGGAA-3 \\
\hline (Muscle atrophy F-Box) & Rev 5'- TTGCAAAGCTGCAGGGTGAC-3' \\
\hline MuRF1 & For 5'-GTGAAGTTGCCCССТTACAA-3' \\
\hline (Muscle RING Finger-1) & Rev 5'-TGGAGATGCAATTGCTCAGT-3' \\
\hline SDHB & For 5'-ATCGAGGACCGGGAGAAG-3' \\
\hline (Succinate dehydrogenase complex, subunit B) & Rev 5'- TTGTCTCCGTTCCACCAGTA-3' \\
\hline \multirow[t]{2}{*}{ Beta-actin } & For 5'- TCTGTGTGGATTGGTGGCTCTA-3' \\
\hline & Rev 5'-CTGCTTGCTGATCCACATCTG-3' \\
\hline \multirow[t]{2}{*}{ Beta-actin (nuclear DNA) } & For 5'-GGGATGTTTGCTCCAACCAA-3' \\
\hline & Rev 5'-GCGCTTTTGACTCAAGGATTTAA-3' \\
\hline
\end{tabular}

Table 3. Characteristics of 23-month-old control and diet-restricted rats

\begin{tabular}{lrr}
\hline & Control & Restricted \\
\hline Body composition $(g)$ & & \\
Initial body weight & $576 \pm 16$ & $577 \pm 29$ \\
Final body weight & $624 \pm 24$ & $375 \pm 13^{\text {a) }}$ \\
Final fat mass & $145 \pm 16$ & $7 \pm 5^{\text {a) }}$ \\
Final lean mass & $451 \pm 12$ & $341 \pm 12^{\text {a) }}$ \\
Tissue mass $(g)$ & & \\
Hindlimb skeletal muscle & $12.28 \pm 0.79$ & $10.10 \pm 0.52^{\text {a) }}$ \\
Liver & $13.94 \pm 0.55$ & $7.52 \pm 0.18^{\text {a) }}$ \\
Heart & $1.60 \pm 0.09$ & $1.28 \pm 0.06^{\text {a) }}$ \\
\hline
\end{tabular}

Data are expressed as means $\pm \operatorname{SEM}(n=10)$.

a) Statistically different from control old rats at $p<0.05$.

\subsection{Statistical analysis}

All data are presented as means \pm SEM. An unpaired Student's $t$-test was performed to test the effect of malnutrition, and one-way ANOVA was used to discriminate the effects of refeeding diets. When the ANOVA indicated significant differences, a post-hoc Dunnett's test was used to identify differences between individual means. Statistical analysis was performed using StatView (version 4.02; Abacus Concepts, Berkeley, CA). Values of $p<0.05$ were considered significant.

\section{Results}

\subsection{Mortality and characterization of body composition and muscle metabolism in old undernourished rats}

There was no difference in mortality observed between control rats and diet-restricted rats during the 3-month dietary restriction period ( $9 \%$ for both groups). At the beginning of the study, there was no significant between-group difference in initial body weight (Table 3). After 3 months of experimentation, undernourished rats had lost $35 \%$ of their initial body weight (Table $3, p<0.05$ ) whereas control rats showed no significant change in body weight. Thus, the diet-restricted group had lower fat mass but also a lower lean mass than age-matched control rats $(-96 \%$ and $-27 \%$ versus control rats, $p<0.05$, respectively) (Table 3). Compared to controls, dietrestricted rats have significantly lower liver mass $(-46 \%$, $p<0.05$ ) and significantly lower muscle mass (cardiac muscle $-20 \%, p<0.05$ and skeletal muscle $-18 \%, p<0.05$ ) (Table 3 ).

FSR of mixed proteins was reduced in plantaris muscle of diet-restricted rats compared to controls $(-18 \%, p<0.05)$ (Fig. 1). FSR of myofibrillar, sarcoplasmic, and mitochondrial proteins decreased to the same extent $(-15$ to $-18 \%)$ as the FSR of total proteins during the dietary restriction period, but only the decrease in mitochondrial protein FSR reached significance (Fig. 1). In addition, p70 S6 kinase and 4EBP1 phosphorylation states were reduced $(-81 \%$ and $-53 \%, p<0.05$, respectively) whereas eIF $2 \alpha$ phosphorylation was increased $(+45 \%, p<0.05)$ in plantaris muscle of undernourished rats compared to controls (Fig. 1). The results on MAFbx and MuRF1 gene expression in plantaris muscle (Fig. 1) indicate a significant increase in MuRF1 mRNA levels in the muscle of diet-restricted rats compared to controls $(+33 \%$, $p<0.05)$ whereas MAFbx mRNA content was not significantly different between groups.

Mitochondrial DNA content and citrate synthase activity were significantly reduced in plantaris muscle of dietrestricted rats compared to controls $(-35 \%$ and $-30 \%$, respectively) (Fig. 2). Consistent with these results, a 3-month dietary restriction period decreased muscle complex II activity by the same extent $(-37 \%, p<0.05)$, possibly due to a significant reduction in complex II subunit gene expression, as observed for SDHB (-28\%, Fig. 2).

\subsection{Similar effect of different high-protein refeeding diets on body composition and tissue mass}

After the dietary restriction period, 23-month old rats were refed ad libitum for 4 weeks with high-protein diets containing $22 \%$ casein (CAS) or $22 \%$ soluble milk proteins (PRO). Spontaneous daily intake was similar for both diets (data not shown). After the 4-week refeeding program, CAS and PRO diet-refed rats showed a similar significant weight gain 
A
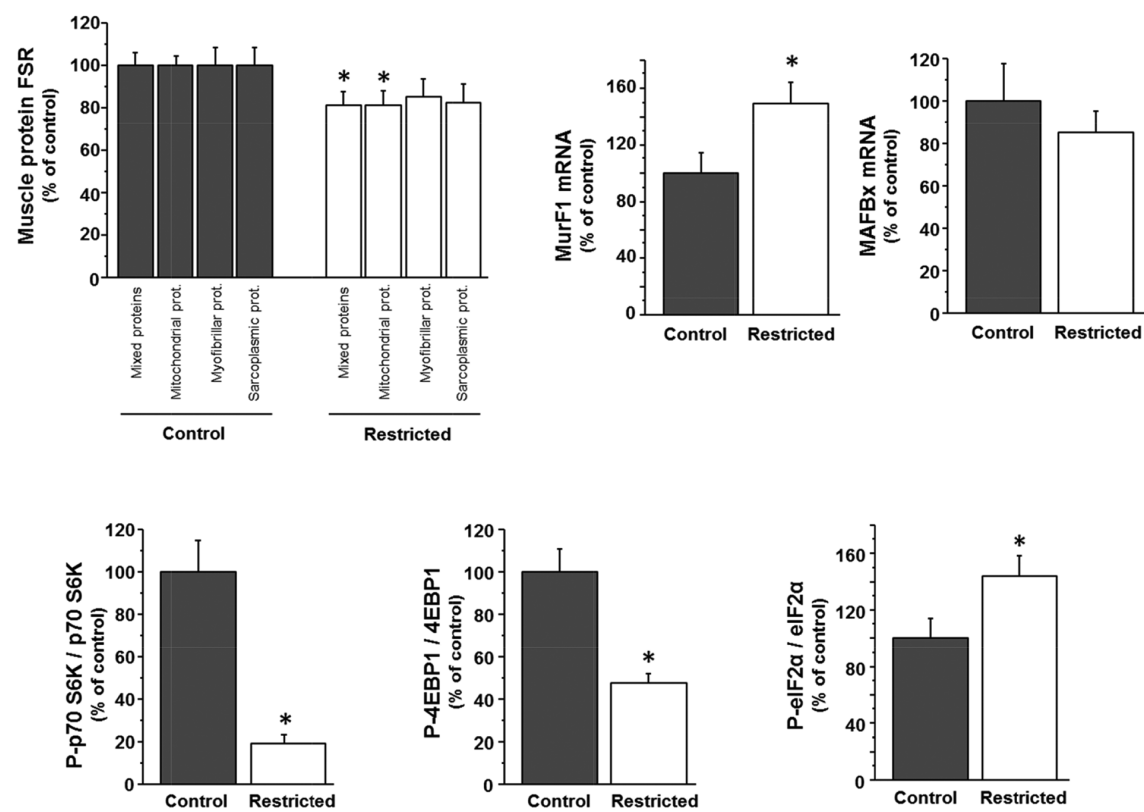

A

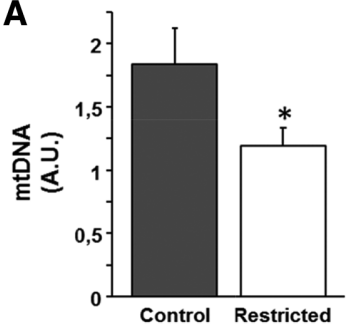

B

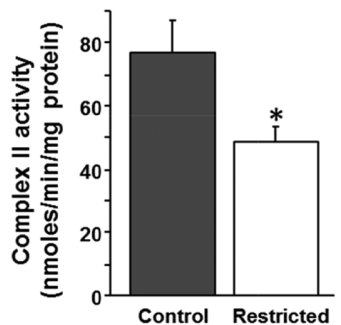

C

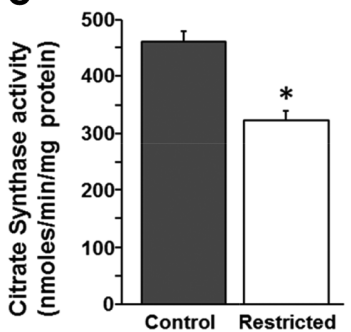

D

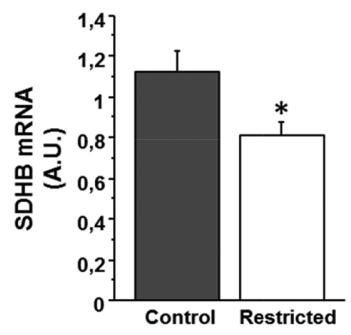

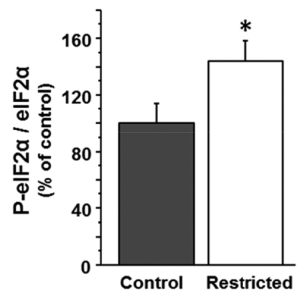

Figure 2. Mitochondrial density (A), enzyme activities (B and C), and complex II subunit transcript levels (D) in the plantaris muscle of malnourished old rats. Twenty-month-old male Wistar rats ( $n=10$ per group) were fed $50 \%$ of their spontaneous intake for 12 weeks to induce malnutrition. Data are presented as means \pm SEM. Unpaired $t$-tests were performed. ${ }^{*} p<0.05$ versus control rats. mtDNA, mitochondrial DNA; SDHB, succinate dehydrogenase complex, subunit $B$.

( $+158 \mathrm{~g}$ and $+169 \mathrm{~g}$ versus diet-restricted rats, respectively) (Fig. 3). Body fat mass was increased in both rat groups compared to undernourished rats, with no between-group differences $(+1040 \%$ and $+1100 \%, p<0.05$, respectively). Likewise, both CAS and PRO diet-refed rats showed a similar significant gain in body lean mass compared to diet-restricted

Figure 1. Protein synthesis rate of different protein fractions and proteolysis markers $(A)$ and translational regulatory pathways $(B)$ in the plantaris muscle of malnourished old rats. Twenty-month-old male Wistar rats ( $n=10$ per group) were fed $50 \%$ of their spontaneous intake for 12 weeks to induce malnutrition. Data are presented as means \pm SEM. Unpaired $t$-tests were performed. ${ }^{*} p<0.05$ versus control rats. FSR, fractional synthesis rate.
B
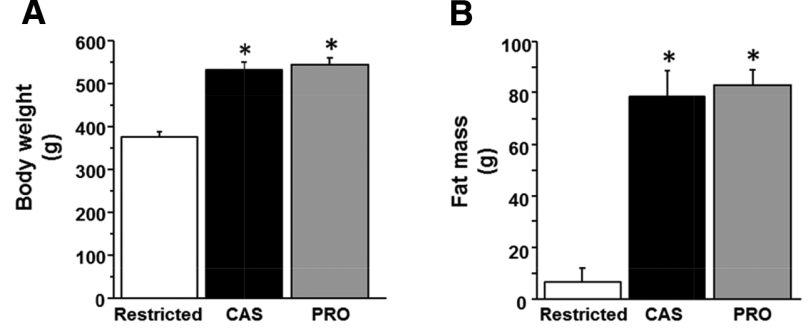

C

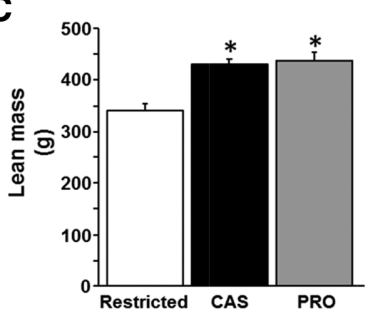

D

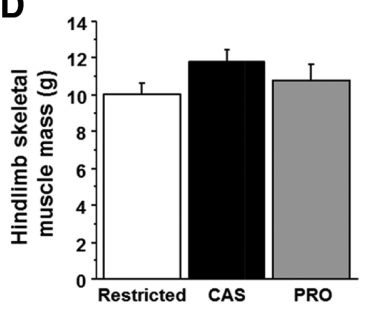

Figure 3. Body weight (A), fat mass (B), lean mass (C), and hindlimb skeletal muscle mass (D) after refeeding with a standard diet enriched with $22 \%$ casein or $22 \%$ soluble milk proteins for 4 weeks in malnourished old rats. Twenty-month-old male Wistar rats ( $n=10$ per group) were fed $50 \%$ of their spontaneous intake for 12 weeks to induce malnutrition. Then, malnourished rats were refed ad libitum with a standard diet enriched with $22 \%$ casein or $22 \%$ soluble milk proteins for 4 weeks. Data are presented as means \pm SEM. ANOVA and post hoc tests were performed. ${ }^{*}<0.05$ versus malnourished old rats. CAS, casein; PRO, soluble milk proteins.

rats ( $+26 \%$ and $+28 \%, p<0.05$, respectively) (Fig. 3). In contrast, hindlimb skeletal muscle mass did not change after 4 weeks of refeeding, regardless of protein quality (Fig. 3). 
Table 4. Protein synthesis rate of total mixed proteins and of different protein fractions in the plantaris muscle of old diet-restricted rats and old diet-restricted rats refed with CAS and PRO diets

\begin{tabular}{llrr}
\hline & Restricted & CAS & PRO \\
\hline FSR (\% of restricted rats) & & & \\
$\quad$ Mixed proteins & $100.00 \pm 7.52$ & $109.18 \pm 5.93$ & $\left.153.14 \pm 11.15^{\mathrm{a})}, \mathrm{b}\right)$ \\
$\quad$ Mitochondrial proteins & $100.00 \pm 8.43$ & $96.81 \pm 6.99$ & $\left.141.50 \pm 10.96^{\mathrm{a})}, \mathrm{b}\right)$ \\
$\quad$ Myofibrillar proteins & $100.00 \pm 9.43$ & $115.13 \pm 5.27$ & $\left.151.77 \pm 5.64^{\mathrm{a}}, \mathrm{b}\right)$ \\
$\quad$ Sarcoplasmic proteins & $100.00 \pm 10.10$ & $118.60 \pm 10.35$ & $150.35 \pm 5.71^{\mathrm{a}), \mathrm{b})}$ \\
\hline
\end{tabular}

Data are expressed as percentage of diet-restricted rat values \pm SEM $(n=8-10)$.

a) Statistically different from diet-restricted old rats at $p<0.05$.

b) Statistically different from diet-restricted CAS diet-refed old rats at $p<0.05$.

\subsection{Refeeding diet supplemented with $22 \%$ soluble milk proteins improved muscle protein synthesis in undernourished old rats}

Mixed, mitochondrial, myofibrillar, and sarcoplasmic protein FSR were increased in the plantaris muscle of PRO diet-refed rats compared to diet-restricted rats $(+41.5-53 \%)$ (Table 4$)$. This increase in muscle protein synthesis in all protein fractions was not observed with the CAS refeeding diet (Table 4). p70 S6 kinase and 4EBP1 phosphorylation states were significantly increased and eIF $2 \alpha$ phosphorylation state was significantly reduced by the refeeding diets, with no significant protein quality effect (Fig. 4A). Of note, fasting blood insulin was increased in refed rats receiving the PRO diet $(0.874$ $\pm 0.105 \mathrm{ng} / \mathrm{mL})$ compared to both CAS diet-refed $(0.585 \pm$ $0.054 \mathrm{ng} / \mathrm{mL}, p<0.05)$ and control $(0.335 \pm 0.038 \mathrm{ng} / \mathrm{mL}$, $p<0.05)$ rats. In addition, CAS and PRO diets significantly reduced MuRF1 gene expression in the plantaris muscle of refed old rats compared to diet-restricted rats (Fig. 4B) (CAS $-32 \%, p<0.05$ and PRO $-43 \%, p<0.05$ ). According to our data, refeeding after the 3-month dietary restriction period did not change muscle MAFbx mRNA content (Fig. 4B).

\subsection{Refeeding diet supplemented with $22 \%$ of soluble milk proteins improved muscle mitochondrial function in undernourished old rats}

The CAS diet improved only one of the mitochondrial parameters explored here. As shown in Fig. 5A, CAS diet-fed rats showed a higher muscle citrate synthase activity than
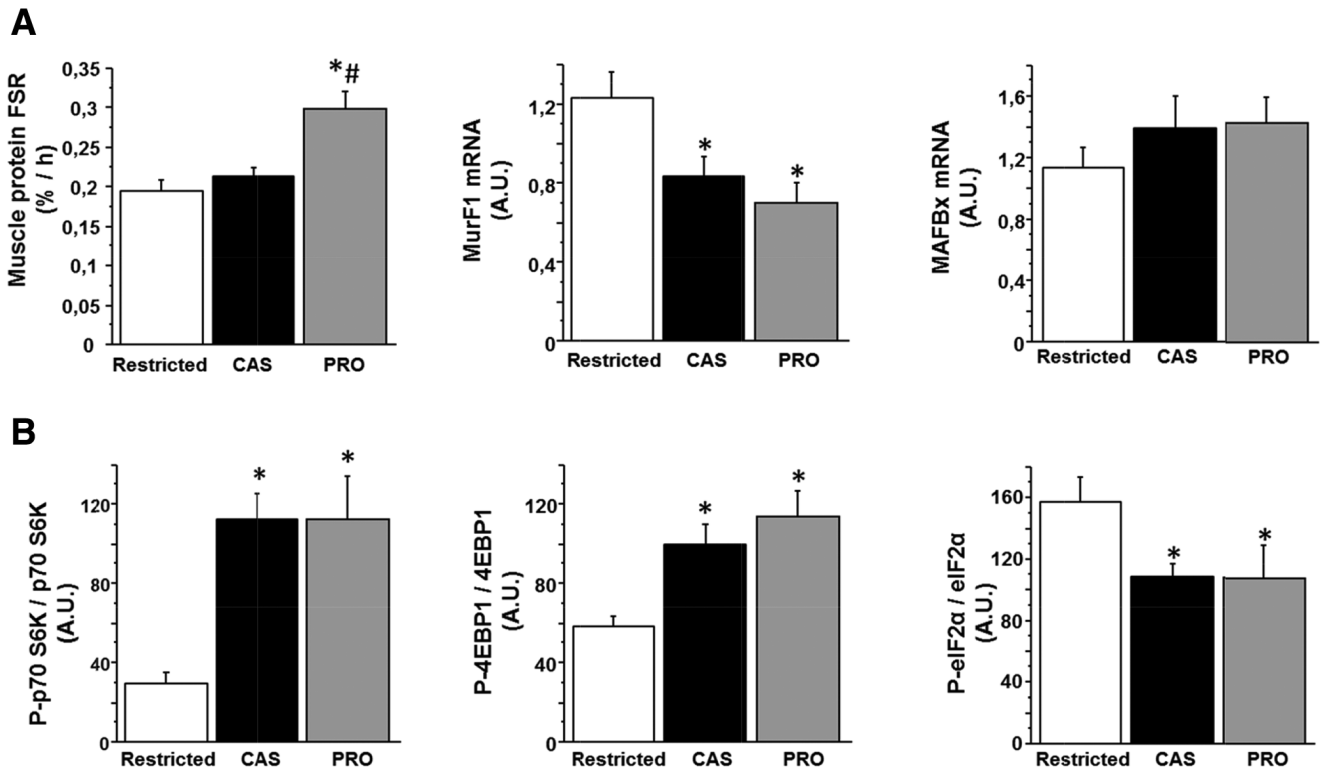

Figure 4. Protein synthesis rate and proteolysis markers $(A)$ and translational regulatory pathways $(B)$ of the plantaris muscle after refeeding with a standard diet enriched with $22 \%$ casein or $22 \%$ soluble milk proteins for 4 weeks in malnourished old rats. Twenty-month-old male Wistar rats ( $n=10$ per group) were fed $50 \%$ of their spontaneous intake for 12 weeks to induce malnutrition. Then, malnourished rats were refed ad libitum with a standard diet enriched with $22 \%$ casein or $22 \%$ soluble milk proteins for 4 weeks. Data are presented as means \pm SEM. ANOVA and post hoc tests were performed. ${ }^{*} p<0.05$ versus malnourished old rats. ${ }^{\#} p<0.05$ versus diet-restricted CAS diet-refed old rats. CAS, casein; PRO, soluble milk proteins; FSR, fractional synthesis rate. 


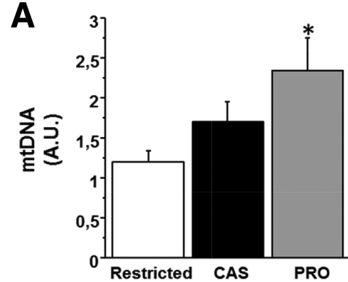

B

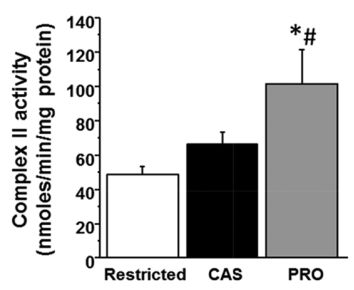

C
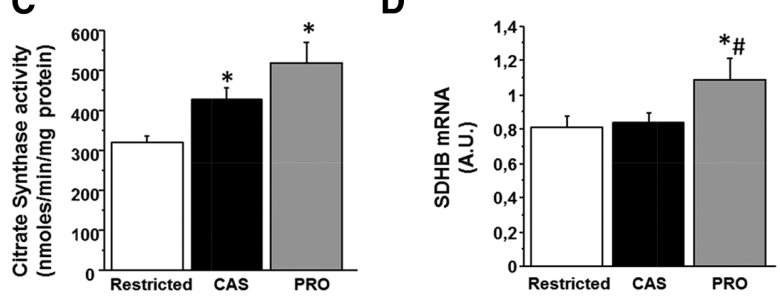

Figure 5. Mitochondrial density (A), enzyme activities (B and $C$ ), and complex 2 subunit transcript levels (D) in the plantaris muscle after refeeding with a standard diet enriched with $22 \%$ casein or $22 \%$ soluble milk proteins for 4 weeks in malnourished old rats. Twenty-month-old male Wistar rats ( $n=10$ per group) were fed $50 \%$ of their spontaneous intake for 12 weeks to induce malnutrition. Then, malnourished rats were refed ad libitum with a standard diet enriched with $22 \%$ casein or $22 \%$ soluble milk proteins for 4 weeks. Data are presented as means \pm SEM. ANOVA and post hoc tests were performed. ${ }^{*} p<0.05$ versus malnourished old rats. ${ }^{\#} p<0.05$ versus diet-restricted CAS diet-refed old rats. CAS, casein; PRO, soluble and native milk proteins; mtDNA, mitochondrial DNA; SDHB, succinate dehydrogenase complex, subunit $B$.

undernourished rats $(+33 \%, p<0.05)$. In contrast, PRO diet improved all the mitochondrial parameters that had been decreased by the dietary restriction period. Mitochondrial DNA content, citrate synthase activity, complex II activity, and SDHB gene expression were all significantly increased in the plantaris muscle of PRO diet-refed rats compared to diet-restricted rats $(+96,+61,+108$, and $+35 \%$, respectively) (Fig. 5).

\section{Discussion}

Undernutrition is the result of a disruption in the equilibrium between dietary intake and nutrient needs. Prolonged undernutrition leads to altered cellular metabolism, loss of body tissues, and, ultimately, impaired body function. Note that here, body weight decreased linearly throughout the experiment in 50\%-food restricted old rats, consistently with our previous observation [16]. These results are also in agreement with Barrows and Roeder [27] who showed in 19-monthold Wistar rats restricted to $50 \%$ of spontaneous food intake that body weight decreased by 34\% after 6 weeks of dietary restriction and failed to stabilize before the death of the animals. As revealed by body composition and tissue mass, protein-energy malnutrition was associated with a significant decrease in both fat mass and lean mass. Hence, the mass of several tissues, including skeletal muscle, was affected in diet-restricted old rats. Clinically, undernutrition is often associated with muscular dysfunction and weakness. In a previous study [28], we showed that young rats adapted to a chronic $50 \%$ dietary restriction by rapid stabilization of body weight, preservation of muscle mass and protein content, and moderate deterioration of nitrogen balance. Unlike adult rats, old rats showed a deficient adaptation to dietary restriction that resulted in severe body and tissue mass loss, depletion of tissue protein content, and deterioration of nitrogen balance $[6,16]$. Here, undernutrition had a significant effect on muscle mass in old rats. Starvation [29] and chronic dietary restriction in weaned and aged rats $[16,30,31]$ were shown to induce a reduction in the amount of protein in skeletal muscle, particularly in fast-twitch muscles [28]. Here, aged undernourished rats showed blunted muscle protein synthesis rates together with increased indexes of muscle proteolysis, i.e. E3 ligase expression, that likely participate in the loss of muscle mass and protein content. To explain this phenomenon, we evaluated the activation of intracellular pathways that upregulate or downregulate muscle protein translation. It appears that some controllers of cellular stress-related pathways that inhibit muscle protein synthesis, i.e. eIF2a [32], were activated in undernourished old rats. In addition, key pathways leading to the activation of muscle protein translation, such as mammalian target of rapamycin (mTOR), were also inhibited in these animals. Note too that the effect of undernutrition on muscle protein synthesis was related to a decreased rate of a specific protein fraction, i.e. mitochondrial proteins. Previous studies suggest that muscle cell energetics are altered by protein-energy restriction, showing altered mitochondrial function and slower rephosphorylation of ADP in the skeletal muscle of malnourished rats [33-35]. Here, and in line with this observation, undernutrition reduced mitochondrial biogenesis and impaired the activity of the Krebs cycle and the activity and gene expression of the mitochondrial electron transport chain in muscle (complex II). Furthermore, another study found that the reduction of complex I-to-III activities was proportional to a reduction in oxidative phosphorylation rate in the muscle of malnourished rats [36].

During the refeeding period, we used two diets containing different sources of proteins, i.e. soluble milk proteins and casein. Other studies have shown that a period of undernutrition induces a decrease in brush border peptidase activity, which in turn may lead to abnormal protein digestion [37]. Holt et al. [13] showed that undernutrition involved a rapid drop in crypt cell proliferation rate that was followed by an abnormal increase in epithelial cell proliferation after refeeding. The high splanchnic extraction of AA during aging might explain the higher intestinal response to refeeding, in particular the increase in cell renewal, and the lower plasma AA concentrations observed in old refed rats compared to younger counterparts. This high splanchnic extraction with increased anabolic threshold is also observed in older people, and seems to decrease with the use of fast-digested proteins, i.e. soluble milk protein [22]. Like casein, soluble milk protein 
is characterized by a high digestive indispensable amino acid score (DIAAS greater than 1). Nevertheless, the nutritional quality of a dietary protein cannot be defined solely on the basis of its digestibility and amino acid composition, especially in pathophysiological situations like aging or undernutrition that induce a loss of body proteins. Other factors should also be considered, such as content of bioactive amino acids or rate of digestion, and soluble milk protein is a better quality protein than casein on both these criteria: soluble milk protein is richer in leucine ( $10 \%$ versus $8 \%$ ) and is more rapidly assimilated. Note too that that both refeeding diets tested here included fat, starch and cellulose that are potentially able to change the digestion kinetics of proteins. However, we previously reported that the other diet components do not influence the digestion kinetics of soluble milk protein and casein $[38,39]$. Concerning the choice of the amount of protein supplementation, based on previous works using equivalent models of old malnourished rats to evaluate the efficacy of refeeding $[15,16,40]$, here we supplied $22 \%$ proteins in the diet to old malnourished rats, which is a significantly higher amount than with standard diet (14\%). Studies in clinical patients $[5,9]$ and old rats [16] have indicated that malnourished older individuals show no response to a diet containing a standard amount of proteins. Also, as early efficacy of renutrition is crucial in elderly people to avoid complications (especially infections), it would be most useful to supply supplements that could act as a kickstarter of renutrition.

Both the whole-body weight and composition of old dietrestricted rats were deeply modified with refeeding. Together with body weight, both fat mass and lean body mass were greater in refed animals compared to their diet-restricted counterparts, regardless of protein source in the refeeding diet. The lack of difference in body weight and composition between refed groups may be because the refeeding period was long enough to enable a restoration of these parameters without a significant difference between dietary protein sources. We previously reported [16] that refeeding old foodrestricted rats with a high-protein diet induced body weight recovery within one week. Here, we show that the increased weight due to refeeding can be explained by an increase in both fat mass and lean mass. Interestingly, changes in body composition were related to changes in the mass of adipose tissues and liver (data not shown). However, note too that despite a sharp increase in muscle protein synthesis rate, hindlimb muscle mass, which had been reduced by dietary restriction, was not significantly improved by refeeding, regardless of protein source. A concomitant increase in proteolysis corresponding to a global increase in muscle protein turnover rate could explain this observation. It has been shown that muscle protein turnover is increased in several cachectic situations and makes it possible to fuel energy needs. This hypothesis was not confirmed by the measure of some simple proteolysis markers, i.e. gene expression of two E3 ligases. Nevertheless, the transcript levels alone of these two enzymes can hardly prefigure the overall activity of the main proteolytic system, i.e. the proteasome-dependent system, and give no indication on other systems. Furthermore, the results of the present study are consistent with our earlier published results on the effect of protein intake level during refeeding in aged rats [16]. In this work, the mass of the tibialis anterior muscle, i.e. one of the hindlimb muscles, was not improved after refeeding with a $23 \%$ protein diet in old diet-restricted rats. Note that in the same study, tibialis anterior mass and protein content in the old group refed with a $27 \%$ protein diet were completely restored and were actually higher than in the group fed the $23 \%$ protein diet during the refeeding period [16]. In addition, as previously shown, muscle function shows an earlier response to refeeding than traditional indicators of muscle mass or muscle composition [41,42], and this same pattern was again found here. We observed a significant improvement in protein turnover capacity and muscle mitochondrial function during the refeeding period, especially when refeeding with the PRO diet, while the mass of muscle tissues remained unchanged. In this context, an important finding was that refeeding with the soluble milk protein diet, but not with the casein diet, restored the protein synthesis rate of mixed muscle proteins as well as each protein fraction, i.e. mitochondrial, myofibrillar, and sarcoplasmic proteins. Therefore, as shown in healthy older men $[17,18]$, soluble milk proteins have intrinsic properties that outperformed casein in improving some of the key metabolic parameters in skeletal muscle in undernourished old rats. Soluble milk proteins not only increased the rate of protein synthesis but also improved the function and activity of some protein fractions. For example, after the period of refeeding with soluble milk proteins, there was a significant increase in mitochondrial protein synthesis rate in connection with improved activity of complex II and citrate synthase. An important factor here is that muscle contraction is dependent on contractile proteins, i.e. myofibrillar proteins, and ATP supply, particularly through mitochondrial oxidative function. Hence, the significant effect of soluble milk proteins on these particular metabolic pathways could more effectively improve muscle contractile function during refeeding and reduce the loss of autonomy often observed in older malnourished patients [43]. We previously demonstrated that using soluble milk proteins to improve mitochondrial and myofibrillar protein synthesis can enhance mitochondrial activity and ATP production and muscle strength in well-nourished old rats [22,44]. We concluded that maintaining protein intake with soluble milk proteins in aging rats helps to curb the progression of sarcopenia by optimizing the turnover rates and functions of major proteins in skeletal muscle. Furthermore, a previous study [45] found that rate of myofibrillar protein synthesis was correlated to muscle strength per unit muscle mass in healthy older people. The decline in leg muscle strength per unit leg muscle mass with aging indicates a decline in the efficiency or quality of this muscle group. It is obvious that myofibrillar proteins are key proteins for muscle contraction, and a decline in the rate of myofibrillar protein synthesis is likely to cause a decline in muscle mass and function. Based on this study [45], it was concluded that a decline in rates 
of myofibrillar protein and mitochondrial protein synthesis provide a theoretical basis for the sarcopenia of aging.

The specific mechanisms responsible for dietary protein activation of the translational regulators, i.e. p70S6k and 4EBP1, are unknown. Soluble milk proteins are known to have a high LEU content, and the use of soluble milk proteins was found to increase plasma LEU concentration. LEU has been shown to exert its effect on muscle protein translation rate through phosphorylation of p70S6k and 4EBP1 via the serine and threonine protein kinase, mTOR [46]. Through the mTOR-dependent pathway, LEU can also stimulate translation elongation by inhibiting the phosphorylation of eIF $2 \alpha$ [47]. Therefore, a better availability of LEU in skeletal muscle may partly explain the effect of this protein on P70S6k, 4EBP1, and eIF2 $\alpha$ phosphorylation in old refed rats. It has been shown [48] that fasting blood concentrations of LEU were significantly elevated (20\%) in human subjects consuming soluble dairy protein. In this study, fasting LEU concentration was positively correlated with lean body mass. A modest increase in basal LEU availability is surely a much weaker anabolic signal than transient postprandial surges, but the total exposure of skeletal muscle to slightly elevated leucine over a 24-hour period could have a role in the adaptive response to refeeding in old rats. Furthermore, rats refed with soluble milk proteins were characterized by higher fasting blood insulin concentrations than rats refed with casein, likely because soluble milk proteins contains amino acids with insulin secretagogue action. Therefore, the conjunction of a possible increase in LEU cell pools with increased insulin might explain the stronger postabsorptive muscle protein synthesis in rats refed with soluble milk proteins. In addition, LEU supplementation restored the postprandial inhibition of in vitro proteasome-dependent proteolysis in 22-month-old animals by downregulating both ubiquitin-conjugating enzyme activities and proteasome activities [49]. Thus, decreased MuRF1 expression in skeletal muscle, as found here, may also be due to increased LEU status in old refed rats. Note however that we observed the same results for effect of refeeding on muscle regulatory pathways whatever the dietary protein considered. Casein contains much less LEU than soluble milk proteins and does not have the same assimilation kinetics as it is absorbed at a slower rate. Also, the upregulatory effect of casein on the mTOR pathway could be offset from its stimulating effect on protein synthesis per se. There would therefore be a disconnect between the effect of casein on the mTOR pathway and its effect on protein synthesis, meaning that other mechanisms may explain the effect of the milk proteins on these regulating pathways in refed old rats. For example, the significant increase in blood insulin level in the CAS group may contribute to the increased phosphorylation of the mTOR pathway. In addition, in the fact that the mTOR signal did not translate into a significant increase in protein synthesis in CAS-refed rats could also be due to cell energy depletion. It has been shown that muscle protein synthesis rate was inversely associated with the phosphorylation states of the "cellular energy sensor" adenosine monophosphate-activated protein kinase- $\alpha$ (AMPK $\alpha)$ and increases in the AMP-to-ATP ratio [50]. The authors concluded that the incongruity between muscle protein synthesis and mTOR activation reflected a block in translation elongation due to reduced cellular energy. Other discordances between muscle protein synthesis and mTOR signaling led the authors to conclude that increasing amino acid and insulin availability causes changes in anabolic signaling, e.g. mTOR signaling, which cannot be easily reconciled with observed effects on muscle protein synthesis rate $[51,52]$.

Besides an effect on muscle protein synthesis rate, refeeding with soluble milk proteins led to a specific increase in muscle mitochondrial function in old malnourished rats. Of note, as observed for protein synthesis, this effect was only seen with the soluble milk proteins. We focused on mitochondria due to its increasingly important role in explaining agingrelated loss of muscle mass and strength, particularly during malnutrition $[22,53]$. We observed that the PRO refeeding diet triggered an increase in complex 2 and CS activities in the plantaris muscle of food-restricted old rats. CS is a key enzyme of the Krebs cycle, and CS activity, besides its functional importance for mitochondria, is also an index of mitochondrial density, together with mitochondrial DNA content $[22,53]$. Thus, refeeding with soluble milk protein-containing diets appears to increase mitochondrial biogenesis in old malnourished rats. Accordingly, complex II activity of the electron transport chain was improved and complex II subunit gene expression was increased, as observed for SDHB. Previous studies [53-55] have highlighted that muscle mitochondrial capacity is dependent not only on mitochondrial density but also on the existence of qualitative differences in mitochondrial functioning. Building on our previous work [56], we propose that the improved mitochondrial functionality with soluble milk protein-supplemented diet may be the result of increased oxidant scavenging and decreased cellular oxidative damage, all of which could be expected to help maintain the functional integrity of the mitochondrial machinery. It is likely, due to an abundance in sulfur AA such as cysteine, that the soluble milk proteins used here demonstrated substantial antioxidant activity. Mice fed on diets containing 20\% soluble milk proteins showed a greater ability to stimulate the synthesis of liver glutathione, which is a cysteine-containing tripeptide, compared to mice fed a $20 \%$ casein diet [57]. Furthermore, it was recently suggested that soluble milk proteins could be used as natural biofunctional ingredients to enhance the antioxidant properties of food products [58]. Increased blood glutathione status has also been observed in healthy humans as well as infection or cancer patients given soluble milk proteins [59-61]. Increased antioxidant defense is consistent with the idea that this particular dietary protein decreases oxidative damage to tissues, thereby preserving the function of cells and cellular components, including mitochondria. Note that several reports have underlined the pivotal role of mitochondrial complex 2 activity in the aging process and in the effect of antioxidant strategies $[62,63]$. In the present study, we were unable to measure the effect 
of soluble milk protein supplementation on glutathione status in old malnourished rats. This is undoubtedly a limit to our work, and further studies are needed to measure the antioxidant effect of soluble milk proteins during renutrition in older subjects. Note, however, that the systemic inflammatory markers measured here (data not shown) were not altered in old rats refed soluble milk proteins compared to their casein-fed counterparts.

In summary, dietary soluble milk proteins have previously been shown to stimulate muscle protein metabolism and contractile function in healthy older people due to their high assimilation rate and their ability to increase amino acid bioavailability. Here we report that supplementing old malnourished rats with soluble milk protein improved the metabolic ability of the skeletal muscle, particularly in terms of protein turnover and mitochondrial oxidative capacities. The abundance of LEU in soluble milk proteins is likely one of the potential mechanisms leading to increased protein synthesis and decreased proteolysis in the skeletal muscle of old malnourished rats. Soluble milk proteins may also represent a safe and effective cysteine donor for glutathione replenishment during glutathione depletion, as observed in malnourished patients, thus contributing to better mitochondrial activity, particularly in skeletal muscle. Taken together, these data argue that soluble milk proteins could play a useful role in the prevention of or recovery from malnutrition in older subjects. Further clinical studies are warranted in order to elucidate the exact mechanisms underpinning these beneficial biological properties and to determine the functional significance of these findings.

This study was supported by grant funding from the French National Agency for Research (ANR).

Jérôme Salles, Audrey Chanet, Alexandre Berry, Christophe Giraudet, Véronique Patrac, Carla Domingues-Faria, Christophe Rocher, Christelle Guillet, Philippe Denis, Patrice Lebecque, and Corinne Pouyet have no conflict of interest to declare.

Cécile Bonhomme and Pascale Le Ruyet are employees of Lactalis Nutrition Santé.

Yves Rolland, Yves Boirie, and Stéphane Walrand received products, i.e. proteins, from Lactalis Nutrition Santé.

\section{References}

[1] Cederholm, T., Nouvenne, A., Ticinesi, A., Maggio, M. et al., The role of malnutrition in older persons with mobility limitations. Curr. Pharm. Des. 2014, 20, 3173-3177.

[2] Morley, J. E., Vellas, B., van Kan, G. A., Anker, S. D. et al., Frailty consensus: a call to action. J. Am. Med. Dir. Assoc. 2013, 14, 392-397.

[3] Guyonnet, S., Rolland, Y., Screening for malnutrition in older people. Clin. Geriatr. Med. 2015, 31, 429-437.

[4] Sullivan, D. H., Walls, R. C., Bopp, M. M., Protein-energy undernutrition and the risk of mortality within one year of hospital discharge: a follow-up study. J. Am. Geriatr. Soc. 1995, 43, 507-512.
[5] Schneider, S. M., Al-Jaouni, R., Pivot, X., Braulio, V. B. et al., Lack of adaptation to severe malnutrition in elderly patients. Clin. Nutr. 2002, 21, 499-504.

[6] Chambon-Savanovitch, C., Felgines, C., Farges, M. C., Pernet, P. et al., Severe dietary restriction initiated in aged rats: evidence for poor adaptation in terms of protein metabolism and intestinal functions. Eur. J. Clin. Invest. 1999, 29, 504511.

[7] Jeejeebhoy, K. N., Malnutrition, fatigue, frailty, vulnerability, sarcopenia and cachexia: overlap of clinical features. Curr. Opin. Clin. Nutr. Metab. Care. 2012, 15, 213-219.

[8] Gordon, B. S., Kelleher, A. R., Kimball, S. R., Regulation of muscle protein synthesis and the effects of catabolic states. Int. J. Biochem. Cell Biol. 2013, 45, 2147-2157.

[9] Hebuterne, X., Schneider, S., Peroux, J. L., Rampal, P. Effects of refeeding by cyclic enteral nutrition on body composition: comparative study of elderly and younger patients. Clin. Nutr. 1997, 16, 283-289.

[10] Boirie, Y., Gachon, P., Beaufrere, B., Splanchnic and wholebody leucine kinetics in young and elderly men. Am. J. Clin. Nutr. 1997, 65, 489-495.

[11] Volpi, E., Sheffield-Moore, M., Rasmussen, B. B., Wolfe, R. R. Basal muscle amino acid kinetics and protein synthesis in healthy young and older men. JAMA 2001, 286, 12061212.

[12] Jourdan, M., Cynober, L., Moinard, C., Blanc, M. C. et al., Splanchnic sequestration of amino acids in aged rats: in vivo and ex vivo experiments using a model of isolated perfused liver. Am. J. Physiol. Regul. Integr. Comp. Physiol. 2008, 294, R748-R755.

[13] Holt, P. R., Yeh, K. Y., Kotler, D. P., Altered controls of proliferation in proximal small intestine of the senescent rat. Proc. Natl. Acad. Sci. USA 1988, 85, 2771-2775.

[14] Poullain, M. G., Cezard, J. P., Marche, C., Macry, J. et al., Effects of dietary whey proteins, their peptides or aminoacids on the ileal mucosa of normally fed and starved rats. Clin. Nutr. 1991, 10, 49-54.

[15] Poullain, M. G., Cezard, J. P., Marche, C., Roger, L. et al., Dietary whey proteins and their peptides or amino acids: effects on the jejunal mucosa of starved rats. Am. J. Clin. Nutr. 1989, 49, 71-76.

[16] Walrand, S., Chambon-Savanovitch, C., Felgines, C., Chassagne, J. et al., Aging: a barrier to renutrition? Nutritional and immunologic evidence in rats. Am. J. Clin. Nutr. 2000, $72,816-824$

[17] Gryson, C., Walrand, S., Giraudet, C., Rousset, P. et al., “Fast proteins" with a unique essential amino acid content as an optimal nutrition in the elderly: growing evidence. Clin. Nutr. 2014, 33, 642-648.

[18] Walrand, S., Gryson, C., Salles, J., Giraudet, C. et al., Fastdigestive protein supplement for ten days overcomes muscle anabolic resistance in healthy elderly men. Clin. Nutr. 2015, 35, 660-668.

[19] Rieu, I., Balage, M., Sornet, C., Giraudet, C. et al., Leucine supplementation improves muscle protein synthesis in elderly men independently of hyperaminoacidaemia. J. Physiol. 2006, 575, 305-315. 
[20] Buford, T. W., Anton, S. D., Judge, A. R., Marzetti, E. et al., Models of accelerated sarcopenia: critical pieces for solving the puzzle of age-related muscle atrophy. Ageing Res. Rev. 2010, 9, 369-383.

[21] Murakami, H., Guillet, C., Tardif, N., Salles, J. et al., Cumulative 3-nitrotyrosine in specific muscle proteins is associated with muscle loss during aging. Exp. Gerontol. 2012, 47, 129135.

[22] Zangarelli, A., Chanseaume, E., Morio, B., Brugere, C. et al., Synergistic effects of caloric restriction with maintained protein intake on skeletal muscle performance in 21-month-old rats: a mitochondria-mediated pathway. FASEB J. 2006, 20, 2439-2450.

[23] Shavlakadze, T., Anwari, T., Soffe, Z., Cozens, G. et al., Impact of fasting on the rhythmic expression of myogenic and metabolic factors in skeletal muscle of adult mice. Am. J. Physiol. Cell. Physiol. 2013, 305, C26-C35.

[24] Benard, G., Faustin, B., Galinier, A., Rocher, C. et al., Functional dynamic compartmentalization of respiratory chain intermediate substrates: implications for the control of energy production and mitochondrial diseases. Int. J. Biochem. Cell Biol. 2008, 40, 1543-1554.

[25] Medja, F., Allouche, S., Frachon, P., Jardel, C. et al., Development and implementation of standardized respiratory chain spectrophotometric assays for clinical diagnosis. Mitochondrion 2009, 9, 331-339.

[26] Gutierrez Cortes, N., Pertuiset, C., Dumon, E., Borlin, M. et al., Novel mitochondrial DNA mutations responsible for maternally inherited nonsyndromic hearing loss. Hum. Mutat. 2012, 33, 681-689.

[27] Barrows, C. H., Jr., Roeder, L. M., The effect of reduced dietary intake on enzymatic activities and life span of rats. $J$. Gerontol. 1965, 20, 69-71.

[28] Felgines, C., Savanovitch, C., Farges, M. C., Cynober, L. et al., Protein metabolism in rats during long-term dietary restriction: influence of aging. JPEN. J. Parenter. Enteral Nutr. 1999, 23, 32-37.

[29] Li, J. B., Higgins, J. E., Jefferson, L. S., Changes in protein turnover in skeletal muscle in response to fasting. Am. J. Physiol. 1979, 236, E222-E228.

[30] el Haj, A. J., Lewis, S. E., Goldspink, D. F., Merry, B. J. et al., The effect of chronic and acute dietary restriction on the growth and protein turnover of fast and slow types of rat skeletal muscle. Comp. Biochem. Physiol. A Comp. Physiol. $1986,85,281-287$.

[31] Goldspink, D. F., el Haj, A. J., Lewis, S. E., Merry, B. J. et al., The influence of chronic dietary intervention on protein turnover and growth of the diaphragm and extensor digitorum longus muscles of the rat. Exp. Gerontol. 1987, 22, 67-78.

[32] Tardif, N., Salles, J., Guillet, C., Tordjman, J. et al., Muscle ectopic fat deposition contributes to anabolic resistance in obese sarcopenic old rats through elF2alpha activation. $\mathrm{Ag}$ ing Cell 2014, 13, 1001-1011.

[33] Briet, F., Jeejeebhoy, K. N., Effect of hypoenergetic feeding and refeeding on muscle and mononuclear cell activities of mitochondrial complexes I-IV in enterally fed rats. Am. J. Clin. Nutr. 2001, 73, 975-983.

[34] Mijan de la Torre, A., Madapallimattam, A., Cross, A., Armstrong, R. L. et al., Effect of fasting, hypocaloric feeding, and refeeding on the energetics of stimulated rat muscle as assessed by nuclear magnetic resonance spectroscopy. J. Clin. Invest. 1993, 92, 114-121.

[35] Pichard, C., Jeejeebhoy, K. N., Muscle dysfunction in malnourished patients. Q. J. Med. 1988, 69, 1021-1045.

[36] Madapallimattam, A. G., Law, L., Jeejeebhoy, K. N., Effect of hypoenergetic feeding on muscle oxidative phosphorylation and mitochondrial complex I-IV activities in rats. Am. J. Clin. Nutr. 2002, 76, 1031-1039.

[37] Boza, J., Jimenez, J., Baro, L., Martinez, O. et al., Effects of native and hydrolyzed whey protein on intestinal repair of severely starved rats at weaning. J. Pediatr. Gastroenterol. Nutr. 1996, 22, 186-193.

[38] Dangin, M., Boirie, Y., Garcia-Rodenas, C., Gachon, P. et al., The digestion rate of protein is an independent regulating factor of postprandial protein retention. Am. J. Physiol. Endocrinol. Metab. 2001, 280, E340-E348.

[39] Dangin, M., Boirie, Y., Guillet, C., Beaufrere, B. Influence of the protein digestion rate on protein turnover in young and elderly subjects. J. Nutr. 2002, 132, 3228S-3233S.

[40] Chyun, J. H., Griminger, P., Improvement of nitrogen retention by arginine and glycine supplementation and its relation to collagen synthesis in traumatized mature and aged rats. J. Nutr. 1984, 114, 1697-1704.

[41] Rigaud, D., Moukaddem, M., Cohen, B., Malon, D. et al., Refeeding improves muscle performance without normalization of muscle mass and oxygen consumption in anorexia nervosa patients. Am. J. Clin. Nutr. 1997, 65, 1845-1851.

[42] Russell, D. M., Leiter, L. A., Whitwell, J., Marliss, E. B. et al., Skeletal muscle function during hypocaloric diets and fasting: a comparison with standard nutritional assessment parameters. Am. J. Clin. Nutr. 1983, 37, 133-138.

[43] Goisser, S., Schrader, E., Singler, K., Bertsch, T. et al., Malnutrition according to mini nutritional assessment is associated with severe functional impairment in geriatric patients before and up to 6 months after hip fracture. J. Am. Med. Dir. Assoc. 2015, 16, 661-667.

[44] Walrand, S., Zangarelli, A., Guillet, C., Salles, J. et al., Effect of fast dietary proteins on muscle protein synthesis rate and muscle strength in ad libitum-fed and energy-restricted old rats. Br. J. Nutr. 2011, 106, 1683-1690.

[45] Balagopal, P., Rooyackers, O. E., Adey, D. B., Ades, P. A. et al., Effects of aging on in vivo synthesis of skeletal muscle myosin heavy-chain and sarcoplasmic protein in humans. Am. J. Physiol. 1997, 273, E790-E800.

[46] Dillon, E. L., Nutritionally essential amino acids and metabolic signaling in aging. Amino. Acids 2013, 45, 431441.

[47] Proud, C. G., Regulation of mammalian translation factors by nutrients. Eur. J. Biochem. 2002, 269, 5338-5349.

[48] Volek, J. S., Volk, B. M., Gomez, A. L., Kunces, L. J. et al., Whey protein supplementation during resistance training 
augments lean body mass. J. Am. Coll. Nutr. 2013, 32, 122135.

[49] Combaret, L., Dardevet, D., Rieu, I., Pouch, M. N. et al., A leucine-supplemented diet restores the defective postprandial inhibition of proteasome-dependent proteolysis in aged rat skeletal muscle. J. Physiol. 2005, 569, 489-499.

[50] Wilson, G. J., Layman, D. K., Moulton, C. J., Norton, L. E. et al., Leucine or carbohydrate supplementation reduces AMPK and eEF2 phosphorylation and extends postprandial muscle protein synthesis in rats. Am. J. Physiol. Endocrinol. Metab. 2011, 301, E1236-E1242.

[51] Atherton, P. J., Etheridge, T., Watt, P. W., Wilkinson, D. et al., Muscle full effect after oral protein: time-dependent concordance and discordance between human muscle protein synthesis and mTORC1 signaling. Am. J. Clin. Nutr. 2010, 92, 1080-1088.

[52] Greenhaff, P. L., Karagounis, L. G., Peirce, N., Simpson, E. J. et al., Disassociation between the effects of amino acids and insulin on signaling, ubiquitin ligases, and protein turnover in human muscle. Am. J. Physiol. Endocrinol. Metab. 2008, 295, E595-E604.

[53] Walrand, S., Short, K. R., Heemstra, L. A., Novak, C. M. et al., Altered regulation of energy homeostasis in older rats in response to thyroid hormone administration. FASEB J. 2014, 28, 1499-1510.

[54] Gueguen, N., Lefaucheur, L., Fillaut, M., Herpin, P. Muscle fiber contractile type influences the regulation of mitochondrial function. Mol. Cell Biochem. 2005, 276, 15-20.

[55] Gueguen, N., Lefaucheur, L., Fillaut, M., Vincent, A. et al., Control of skeletal muscle mitochondria respiration by adenine nucleotides: differential effect of ADP and ATP according to muscle contractile type in pigs. Comp. Biochem. Physiol. B Biochem. Mol. Biol. 2005, 140, 287-297.

[56] Lanza, I. R., Zabielski, P., Klaus, K. A., Morse, D. M. et al., Chronic caloric restriction preserves mitochondrial function in senescence without increasing mitochondrial biogenesis. Cell Metab. 2012, 16, 777-788.

[57] Pacheco, M. T., Sgarbieri, V. C., Effect of different hydrolysates of whey protein on hepatic glutathione content in mice. J. Med. Food 2005, 8, 337-342.

[58] Mann, B., Kumari, A., Kumar, R., Sharma, R. et al., Antioxidant activity of whey protein hydrolysates in milk beverage system. J. Food Sci. Technol. 2015, 52, 3235-3241.

[59] Bounous, G., Whey protein concentrate (WPC) and glutathione modulation in cancer treatment. Anticancer. Res. 2000, 20, 4785-4792.

[60] Micke, P., Beeh, K. M., Buhl, R., Effects of long-term supplementation with whey proteins on plasma glutathione levels of HIV-infected patients. Eur. J. Nutr. 2002, 41, 12-18.

[61] Zavorsky, G. S., Kubow, S., Grey, V., Riverin, V. et al., An open-label dose-response study of lymphocyte glutathione levels in healthy men and women receiving pressurized whey protein isolate supplements. Int. J. Food Sci. Nutr. 2007, 58, 429-436.

[62] Gredilla, R., Phaneuf, S., Selman, C., Kendaiah, S. et al., Short-term caloric restriction and sites of oxygen radical generation in kidney and skeletal muscle mitochondria. Ann. NY Acad. Sci. 2004, 1019, 333-342.

[63] Ruiz, L. M., Jensen, E. L., Bustos, R. I., Arguelloa, G. et al., Adaptive responses of mitochondria to mild copper deprivation involve changes in morphology, OXPHOS remodeling and bioenergetics. J. Cell. Physiol. 2014, 229, 607-619. 